\title{
Condorcet efficiency of general weighted scoring rules under IAC: indifference and abstention
}

\author{
Mostapha Diss, Eric Kamwa, Issofa Moyouwou, and Hatem Smaoui
}

\begin{abstract}
In an election, individuals may sometimes abstain or report preferences that include ties among candidates. How abstention or ties within individual preferences impact the performances of voting rules is a natural question addressed in the literature. We reconsider this question with respect to one of the main characteristics of a voting rule: its Condorcet efficiency; that is, the conditional probability that the rule selects a Condorcet winner assuming that one exists. We explore the impact of both ties and abstention on the Condorcet efficiency of the whole class of weighted scoring rules in three-candidate elections under the Impartial Anonymous Culture assumption. It appears in general that the possibility of indifference or abstention increases or decreases the Condorcet efficiency of weighted scoring rules depending on the rule under consideration or the probability distribution on the set of observable voting situations.
\end{abstract}

\footnotetext{
Mostapha Diss

CRESE EA3190, Univ. Bourgogne Franche-Comté, F-25000 Besançon, France. e-mail: mostapha.diss@univ-fcomte.fr

Eric Kamwa

LC2S UMR CNRS 8053, Faculté de Droit et d'Economie de la Martinique, Université des Antilles, F-97275 Schœlcher Cedex, France. e-mail: eric.kamwa@univ-antilles.fr.

Issofa Moyouwou

Ecole Normale Supérieure - Department of Mathematics - University of Yaounde I. BP 47 Yaounde, Cameroon, e-mail: imoyouwou2@yahoo.fr

Hatem Smaoui

CEMOI, Université de La Réunion, Saint-Denis, France. e-mail: hatem.smaoui@ univ-reunion.fr.
} 


\section{Introduction}

The simplest representation of a voting environment includes a set of voters, a set of candidates, a list of admissible individual preferences and a rule that aggregates each possible configuration of voters' preferences into a social outcome. In this context, a profile is defined as a sequence of preferences of all the individuals taking part in the vote. The performance of a voting rule is then measured by its propensity to avoid counterintuitive results or to produce desirable electoral outcomes. For example, there may exist a candidate that is preferred to any other candidate by a majority of voters, a Condorcet winner; Condorcet (1875) advocated that when a Condorcet winner exists, he/she should be the outcome of any reasonable rule. But undertaking in practice all pairwise majority comparisons for a given profile of individual preferences is very demanding as the total number of voters or of candidates increases. An earlier alternative suggested by Borda (1781) consists in assigning an amount of points to each candidate each time he/she is ranked at a given position by a voter. For example with three candidates, 1 point is awarded to each candidate for each first place in an individual ranking, $\lambda$ points for each second place where $0 \leq \lambda \leq 1$ and no point for a last position. The winner is then the candidate with the highest total score. However, all such weighted scoring rules may fail to select a Condorcet winner. Since then, the ability of a weighted scoring rule to select a Condorcet winner has been the subject of an abundant literature that aims at measuring the desirability of a voting rule with respect to its Condorcet efficiency; that is, the conditional probability that the rule will select a Condorcet winner assuming that one exists.

Cervone et al. (2005) investigated in three-candidate elections which (oneshot) weighted scoring rule exhibits the maximum Condorcet efficiency under the Impartial Anonymous Culture (IAC) assumption. First explored by Gehrlein and Fishburn (1976), the IAC assumption amounts to assuming that all anonymous profiles of individual preferences are equally probable. Cervone et al. (2005) showed that when individual preferences are linear orders, the weighted scoring rule that performs the best in selecting a Condorcet winner is a rule that lies between the Plurality rule $(\lambda=0)$ and the Borda rule $(\lambda=0.5)$. In this paper, we address a similar question when individual preferences are weak orders (some voters may be indifferent between two or more candidates); or when some voters may abstain (they freely decide to not participate in the election).

The possibility that voter indifference may be observable has already been considered by some other authors; see for example Diss et al. (2010), Gehrlein and Lepelley (2015), Kamwa (2019b), or Merlin and Valognes (2004) among others. More precisely, we propose an IAC counterpart of Gehrlein and Valognes (2001), who considered the same topic, when we assume that each voter uniformly picks his/her preference out of a predefined set of weak orders. This is known as an Impartial Culture (IC)-like probability distribution over the 
set of all configurations of voters' preferences. Here, two extreme cases are explored. We first consider only concerned voters, i.e., voters with strict preferences on at least a pair of candidates. We determine the exact Condorcet efficiency of each possible weighted scoring rule under the IAC assumption in three-candidate elections when the total number of voters tends to infinity. It appears that when we move from a weighted scoring rule with linear orders to its extended version on weak orders, the Condorcet efficiency increases for all weights $\lambda$ ranged from 0 up to approximately 0.3765 ; but decreases for all weights ranged from 0.3765 to 1 . Another salient point is that the maximum Condorcet efficiency in three-candidate elections under the IAC assumption is now observed for a new weight, approximately 0.4139 , which is distinct from the optimal one provided by Cervone et al. (2005) which is approximately 0.3723. Finally, the maximum Condorcet efficiency is now equal to 0.9265 , which is slightly greater than 0.9255 , the one obtained with linear orders.

The possibility that some voters will abstain is also explored, and here we follow the recent framework of Gehrlein and Lepelley (2020, 2017). The authors measured the impact of indifference on voting rules with respect to the participation rate under both IC and IAC assumptions. We explore some new and extreme cases assuming that the participation rate is unknown and may be of any size. Three cases are considered: global abstention when voters from all possible types may abstain; self-confident abstention when only voters who prefer a Condorcet winner by self-confidence abstain - this may presumably be the case when a Condorcet winner exists and is acclaimed by almost all polls; and pessimistic abstention when only voters who prefer any other candidate to a Condorcet winner by discouragement abstain - this may be the case when some voters think their favorite candidate is lagging behind their less preferred candidate. In the global abstention setting, we would have expected a very low Condorcet efficiency for every weighted scoring rule; but in fact we observe an honorable performance, since some weighted scoring rules still record more than $60 \%$ of voting situations in which the Condorcet winner is selected after some voters abstain. The two other cases of abstention impact differently on the performances of weighted scoring rules. All those aspects are commented upon and discussed later in the paper.

The rest of the paper is organized as follows: Section 2 underlines some key points of our investigations that differ from previous works. In Section 3 , for a three-candidate election we provide the exact Condorcet efficiency of any weighted scoring rule as the total number of voters tends to infinity. Section 4 highlights some abstention patterns and the Condorcet efficiency of weighted scoring rules on those restricted domains. Section 5 concludes with a general comment on our investigations. 


\section{The scope}

Consider a three-candidate election with $n$ voters $(n \geq 2)$ and assume that an individual preference is a weak order (complete and transitive binary relations) over candidates $A, B$ and $C$. In addition, each voter is assumed to act according to his/her true preferences, which means that strategic voting is not taken into consideration in our paper. There are thirteen possible types of preferences according to how candidates are ranked with or without indifference:

$$
\begin{aligned}
& A \succ B \succ C\left(x_{1}\right) \quad A \succ(B \sim C) \quad\left(x_{7}\right) \\
& A \succ C \succ B\left(x_{2}\right) \quad B \succ(A \sim C)\left(x_{8}\right) \\
& B \succ A \succ C\left(x_{3}\right) \quad C \succ(A \sim B) \quad\left(x_{9}\right) \\
& B \succ C \succ A\left(x_{4}\right) \quad(A \sim B) \succ C\left(x_{10}\right) \\
& C \succ A \succ B\left(x_{5}\right) \quad(A \sim C) \succ B\left(x_{11}\right) \\
& C \succ B \succ A\left(x_{6}\right) \quad(B \sim C) \succ A\left(x_{12}\right) \\
& (A \sim B \sim C)\left(x_{13}\right)
\end{aligned}
$$

In the notation $A \succ B \succ C\left(x_{1}\right), A \succ B \succ C$ refers to the preference type of all voters who prefer $A$ to $B, A$ to $C$, and $B$ to $C$; and $x_{1}$ is the proportion of such voters; that is the ratio $\frac{n_{1}}{n}$ where $n_{1}$ is the total number of voters who report $A \succ B \succ C$. Similarly, a voter endowed with the preference type $A \succ(B \sim C)$ prefers $A$ to $B, A$ to $C$ and is indifferent between $B$ and $C$. The proportion of all such voters is $x_{7}$. The collection $x=\left(x_{1}, x_{2}, \ldots, x_{13}\right)$ will be called a voting situation when the thirteen terms $x_{j}$ sum to 1 . Voters having the preference type $(A \sim B \sim C)$ will be called unconcerned voters since each such voter is indifferent to the election of any of the three candidates. In case there is some evidence that allows each voter to have a strict ranking of the three competing candidates, only the first six preference types are observable. A voting situation will then be reduced to the 6 -tuple $x=\left(x_{1}, x_{2}, \ldots, x_{6}\right)$ by setting $x_{7}=x_{8}=\ldots=x_{13}=0$. This is the assumption taken into account by Cervone et al. (2005).

Indifference or abstention are possible factors that may justify alternative investigations. Given indifference, we carry out our investigations under two different but common settings. When all voters are concerned voters who may still be indifferent between at most two candidates, we identify a voting situation as the 12 -tuple $x=\left(x_{1}, x_{2}, \ldots, x_{12}\right)$ assuming that $x_{13}=0$. This is also the setting taken into consideration by Gehrlein (1983). We also consider the mixed case where unconcerned and concerned voters are involved. In this latter case, only voting situations $x=\left(x_{1}, x_{2}, \ldots, x_{12}, x_{13}\right)$ such that $0 \leq$ $x_{13}<1$ are considered (the extreme case $x_{13}=1$ removes the possibility of any objective differentiation among the three candidates). This is the setting developed by Gehrlein and Valognes (2001).

When indifference vanishes, some voters may abstain and a voting scenario is now a twofold vector $(x, y)$ where $x=\left(x_{1}, x_{2}, \ldots, x_{6}\right)$ is the initial voting situation and $y=\left(y_{1}, y_{2}, \ldots, y_{6}\right)$ indicates the proportion $y_{j}$ of voters who abstain among the voters having preference of type $j$. Note that $0 \leq y_{j} \leq x_{j}$ 
and $y_{1}+y_{2}+\ldots+y_{6}<1$ (the extreme case $y_{1}+y_{2}+\ldots+y_{6}=1$ is of no interest). The impact of abstention on voting procedures was also studied in Gehrlein and Fishburn $(1978,1979)$ and recently by Gehrlein and Lepelley (2020, 2017). We provide two extreme cases: (i) when all voters who abstain have the same top-ranked candidate (who is perceived as having no chance to win); and (ii) when all voters who abstain have the same bottom-ranked candidate.

In each of the preference settings we consider, our analysis is restricted to the set of voting situations that capture all the possible configurations of individual preferences. We denote this set by $D$ and we assume for each case the uniform probability distribution over $D$ : all voting situations in $D$ are equally probable to be observed. This is known as an Impartial Anonymous Culture assumption over $D$ and will be referred to as $I A C_{D}$. Given $\lambda \in[0,1]$, the vector $w=(1, \lambda, 0)$ will be called the scoring vector. The weighted scoring rule on $D$ is denoted by $F_{\lambda}$ and assigns $x_{j} w(j, k)$ points to a candidate, say $C$, each time voters having type $j$ rank $C$ at the $k^{\text {th }}$ position given a voting situation $x$; where $w(j, k)=w_{k}$ in case preference of type $j$ corresponds to a linear order $(j=1,2, \ldots, 6$ and $k=1,2,3) ; w(j, 1)=\frac{(1+\lambda)}{2}$ and $w(j, 2)=0$ if voters of type $j$ are indifferent between their two first-ranked candidates $(j=7,8,9) ; w(j, 1)=1$ and $w(j, 2)=\frac{\lambda}{2}$ if voters of type $j$ are indifferent between their two bottom-ranked candidates $(j=9,10,11)$; and $w(j, 1)=$ $\frac{(1+\lambda)}{3}$ if $j=13$. Obviously, the candidate who records the maximum sum of points wins.

A candidate $X$ majority defeats another candidate $Y$ in a pairwise comparison if there are more voters who strictly prefer $X$ to $Y$ than voters who strictly prefer $Y$ to $X$. A Condorcet winner is a candidate who defeats any other candidate in pairwise majority voting. When a Condorcet winner exists, he/she is clearly a desirable election winner since he/she is immune to rejection by any majority of voters. It is well known that for a given weighted scoring rule $F_{\lambda}$, we may find some voting situation $x$ in which a candidate, say $C$, is a Condorcet winner while $F_{\lambda}(x) \neq C$. Courtin et al. (2015a) show that this failure may be overcome in three-candidate elections by strengthening the size of the majority in favor of the Condorcet winner up to a threshold; see also Courtin et al. (2015b) for a more general framework.

In general, the Condorcet efficiency of the rule $F_{\lambda}$, given a domain $D$ of observable voting situations with $n$ voters and a probability distribution $P_{D}$ over $D$, is the conditional probability $C E\left(\lambda, P_{D}, n\right)$ that the rule will select a Condorcet winner assuming that one exists. In particular, under the $I A C$ assumption, the limit $C E\left(\lambda, I A C_{D}, \infty\right)$ of $C E\left(\lambda, I A C_{D}, n\right)$ as $n$ tends to infinity is the ratio $\frac{\operatorname{vol}\left(D_{C W, \lambda}\right)}{\operatorname{vol}\left(D_{C W}\right)}$ where $D_{C W}$ denotes the polytope of all voting situations in $D$ in which a Condorcet winner exists ${ }^{1}$ while $D_{C W, \lambda}$ is the polytope of all voting situations in $D$ at which a Condorcet winner exists and is the winner for rule $F_{\lambda}$; for more details and a rich panel of related

\footnotetext{
${ }^{1}$ i.e., the polytope defined by the linear system characterizing these voting situations.
} 
topics, interested readers are referred to Gehrlein (2006) or Gehrlein and Lepelley $(2017,2011)$. By symmetry, to evaluate $C E\left(\lambda, I A C_{D}, \infty\right)$, we can replace $D_{C W}$ by $D_{C W, A}$, the subset of $D_{C W}$ in which $A$ is the Condorcet winner, and $D_{C W, \lambda}$ by $D_{C W, \lambda, A}$, the subset of $D_{C W, \lambda}$ in which $A$ is the Condorcet winner and is selected by the voting rule $F_{\lambda}$.

\section{Condorcet efficiency of weighted scoring rules when indifference is observable}

Giving a weight $\lambda \in[0,1]$, we determine here the Condorcet efficiency of the weighted scoring rule associated with $\lambda$ when some voters may be indifferent between two candidates.

\subsection{With no unconcerned voters}

When no voter is unconcerned and none of them abstains, the corresponding domain of observable voting situations is denoted by $\mathcal{D}$ and consists of all 12-tuples $x=\left(x_{1}, x_{2}, \ldots, x_{12}\right)$ such that

$$
\sum_{j=1}^{12} x_{j}=1 \text { and } x_{j} \geq 0 \text { for all } j \in\{1,2, \ldots, 12\} .
$$

In this case, candidate $A$ is a Condorcet winner in $x$ if $A$ beats $B$ and $A$ beats $C$ in pairwise majority voting:

$$
\begin{aligned}
& x_{3}+x_{4}+x_{6}+x_{8}+x_{12}-x_{1}-x_{2}-x_{5}-x_{7}-x_{11}<0 \\
& x_{4}+x_{5}+x_{6}+x_{9}+x_{12}-x_{1}-x_{2}-x_{3}-x_{7}-x_{10}<0
\end{aligned}
$$

Therefore, the set $\mathcal{D}_{C W, A}$ of all voting situations in $\mathcal{D}$ at which $A$ is the Condorcet winner is the 11-dimensional polytope defined by (1), (2), and (3). The volume of $\mathcal{D}_{C W, A}$ as well as all other subsequent volumes in this paper will be computed using the method presented in Moyouwou and Tchantcho (2017). Alternative methods are also available from Cervone et al. (2005) or Lepelley et al. (2008). We may also combine available packages such as Convex for convex geometry by Franz (2017) for a Maple implementation or well-established algorithms such as Normaliz by Bruns et al. (2017, 2019) and Bruns and Ichim (2010). These techniques have also recently been used under different forms by Bubboloni et al. (2019), Diss and Doghmi (2016), Diss et al. (2018), Diss and Gehrlein (2012, 2015), Kamwa (2019a), Kamwa and Moyouwou (2020), El Ouafdi et al. (2019); Lepelley et al. (2018b), and 
Lepelley and Smaoui (2019), among others. Up to a scaling constant that depends only on the dimension of $\mathcal{D}$,

$$
\operatorname{vol}(\mathcal{D})=\frac{1}{11 !} \text { and } \operatorname{vol}\left(\mathcal{D}_{C W}\right)=3 \operatorname{vol}\left(\mathcal{D}_{C W, A}\right)=\frac{8821}{367873228800}
$$

Then, the probability that a Condorcet winner exists under the assumption $I A C_{\mathcal{D}}$ is $\frac{\operatorname{vol}\left(\mathcal{D}_{C W}\right)}{\operatorname{vol}(\mathcal{D})} \approx 0.95714$. As compared to the probability 0.9375 from Gehrlein and Fishburn (1976) of observing a Condorcet winner when individual preferences are linear orders, this confirms the observation by Gehrlein and Valognes (2001) that the possibility of indifference increases the probability that a Condorcet winner exists. Now candidate $A$ is the winner for rule $F_{\lambda}$ at $x$ when the score of $A$ is greater than both the score of $B$ and the score of $C$; that is

$$
\begin{aligned}
& (\lambda-1)\left(x_{1}-x_{3}\right)-x_{2}+x_{4}-\lambda\left(x_{5}-x_{6}\right)+\frac{\lambda-2}{2}\left(x_{7}-x_{8}\right)-\frac{1+\lambda}{2}\left(x_{11}-x_{12}\right)<0 \\
& -x_{1}+x_{6}+(\lambda-1)\left(x_{2}-x_{5}\right)-\lambda\left(x_{3}-x_{4}\right)+\frac{\lambda-2}{2}\left(x_{7}-x_{9}\right)-\frac{1+\lambda}{2}\left(x_{10}-x_{12}\right)<0
\end{aligned}
$$

The subset $\mathcal{D}_{C W, A, \lambda}$ of $\mathcal{D}_{C W, A}$ that consists of all voting situations in which $A$ is the Condorcet winner and at the same time is selected by $F_{\lambda}$ at $x$ is the polytope described by the constraints at (1), (2), (3), (5), and (6). Its volume is computed as a function of $\lambda$ in order to derive the Condorcet efficiency $C E\left(\lambda, I A C_{\mathcal{D}}, \infty\right)=\frac{\operatorname{vol}\left(\mathcal{D}_{C W, A, \lambda}\right)}{\operatorname{vol}\left(\mathcal{D}_{C W, A}\right)}$ when the total number of voters tends to infinity. The corresponding formula is completely unreadable and is relegated to the Appendix. Numerical values of this function are reported in Table 1 and its graph appears in Figure 1. The value of $C E\left(\lambda, I A C_{\mathcal{D}}, \infty\right)$ provided in Table 1 corresponds to some values of $\lambda=d_{1}+d_{2}$, the first decimal $\left(d_{1}\right)$ of which is indicated in the first column and the second decimal $\left(d_{2}\right)$ in the first row. Moreover, the maximum of $C E\left(\lambda, I A C_{\mathcal{D}}, \infty\right)$ is for a unique value $\lambda^{*}$ of $\lambda$ between $\frac{1}{3}$ and $\frac{1}{2}$. The exact value of $\lambda^{*}$ is unreachable due to the intractable expressions of $C E\left(\lambda, I A C_{\mathcal{D}}, \infty\right)$ and of its first derivative. An approximation up to four decimal places gives $\lambda^{*} \approx 0.4139$ with $C E\left(\lambda^{*}, I A C_{\mathcal{D}}, \infty\right) \approx 0.9265$.

When no voter is indifferent between any pair of candidates, the function of the Condorcet efficiency of all weighted scoring rules with three candidates under the IAC assumption comes from Cervone et al. (2005, Theorem 2). Its graph is also represented in Figure 1. From this result, it appears that the rule that maximizes the Condorcet efficiency among weighted scoring rules corresponds to a value $\lambda_{0}$ of the weight $\lambda$ such that $2 \lambda_{0}-1 \approx-0.25544$; that is $\lambda_{0} \approx 0.37228$. It follows that if we are looking for the optimal weighted scoring rule with respect to Condorcet efficiency under the IAC assumptions described above, the appropriate value of the weight $\lambda$ differs when we admit indifference or only consider linear orders. With indifference, our results show that the optimal rule is nearer to the Borda rule $(\lambda=0.5)$ than it is with only 
Table 1 IAC-based Condorcet efficiency of weighted scoring rules when indifference is observable

\begin{tabular}{|c|c|c|c|c|c|c|c|c|c|c|}
\hline$\lambda$ & 0.00 & 0.01 & 0.02 & 0.03 & 0.04 & 0.05 & 0.06 & 0.07 & 0.08 & 0.09 \\
\hline 0.0 & 0.8575 & 0.8596 & 0.8618 & 0.8640 & 0.8662 & 0.8683 & 0.8705 & 0.8727 & 0.8749 & 0.8770 \\
\hline 0.1 & 0.8792 & 08814 & 0.8835 & 0.8856 & 0.8878 & 0.8899 & 0.8920 & 0.8941 & 0.8961 & 0.8981 \\
\hline 0.2 & 0.9001 & 0.9021 & 0.9040 & 0.9059 & 0.9078 & 0.9095 & 0.9113 & 0.9130 & 0.9146 & 0.9161 \\
\hline 0.3 & 0.9176 & 0.9190 & 0.9202 & 0.9214 & 0.9225 & 0.9235 & 0.9243 & 0.9251 & 0.9256 & 0.9261 \\
\hline 0.4 & 0.9264 & 0.9265 & 0.9265 & 0.9263 & 0.9260 & 0.9254 & 0.9247 & 0.9238 & 0.9227 & 0.9214 \\
\hline 0.5 & 0.9199 & 0.9182 & 0.9163 & 0.9143 & 0.9121 & 0.9097 & 0.9071 & 0.9044 & 0.9016 & 0.8986 \\
\hline 0.6 & 0.8955 & 0.8923 & 0.8889 & 0.8855 & 0.8819 & 0.0100 & 0.8746 & 0.8708 & 0.8669 & 0.8630 \\
\hline 0.7 & 0.8590 & 0.8549 & 0.8508 & 0.8466 & 0.8424 & 0.8382 & 0.8338 & 0.8295 & 0.8251 & 0.8207 \\
\hline 0.8 & 0.8163 & 0.8119 & 0.8074 & 0.8029 & 0.7984 & 0.7939 & 0.7894 & 0.7849 & 0.7803 & 0.7758 \\
\hline 0.9 & 0.7713 & 0.7668 & 0.7622 & 0.7577 & 0.7532 & 0.7487 & 0.7442 & 0.7397 & 0.7353 & 0.7308 \\
\hline 1 & 0.7264 & - & - & - & - & - & - & - & - & - \\
\hline
\end{tabular}

Fig. 1 IAC-based Condorcet efficiency of weighted scoring rules with and without indifference

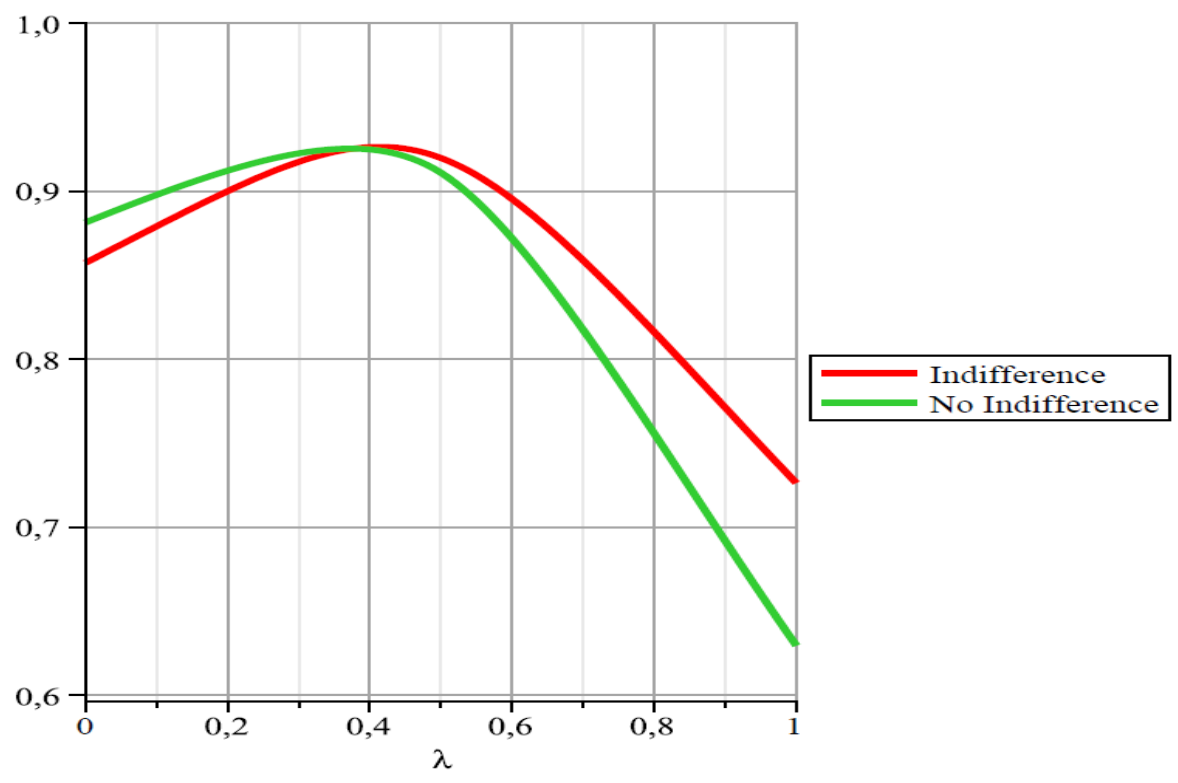

linear orders. Finally it is worth noting from our results that the maximal Condorcet efficiency among weighted scoring rules is approximately 0.9265 . This value is greater than the maximal Condorcet efficiency among weighted scoring rules with only linear orders, which is approximately 0.9255 (Cervone et al., 2005, Theorem 2). Again, this is in accordance with earlier observations. 
However, the Condorcet efficiency of some weighted scoring rules decreases from linear orders to weak orders as shown in Figure 1. More exactly, the Condorcet efficiency is greater for linear orders than for weak orders for all weighted scoring rules from $\lambda=0$ (the Plurality rule) up to $\lambda \approx 0.3765$. Globally, the extensions of classical weighted scoring rules that permit us to handle the possible indifference of voters result in improvements of the Condorcet efficiency of weighted scoring rules for $1 \geq \lambda>0.3765$ but not for $0 \leq \lambda<0.3765$.

\subsection{With possibly unconcerned voters}

When some voters are completely indifferent about the selection of one of the three candidates, the corresponding domain of observable voting situations is denoted by $\widetilde{\mathcal{D}}$ and consists of all 13 -tuples $x=\left(x_{1}, x_{2}, \ldots, x_{13}\right)$ such that

$$
\sum_{j=1}^{13} x_{j}=1 \text { and } x_{j} \geq 0 \text { for all } j \in\{1,2, \ldots, 13\} \text {. }
$$

Such a voting situation is completely determined by the 12 -tuple $\left(x_{1}, x_{2}, \ldots, x_{12}\right)$ which satisfies

$$
\sum_{j=1}^{12} x_{j}=t \text { with } t=1-x_{13}>0 .
$$

More interestingly, the conditions that candidate $A$ is the Condorcet winner or the winner for the weighted scoring rule $F_{\lambda}$ do not change, since an unconcerned voter does not favor any of the three candidates. By setting $x_{j}=t y_{j}$ for $j=1,2, \ldots, 12$, it follows that $A$ is the Condorcet winner at $x$ if and only if $y=\left(y_{1}, y_{2}, \ldots, y_{12}\right)$ lies in $\mathcal{D}_{C W, A}$ characterized by (1), (2) and (3). Similarly $A$ is the winner at $x$ for $F_{\lambda}$ if and only if $y=\left(y_{1}, y_{2}, \ldots, y_{12}\right)$ belongs to $\mathcal{D}_{C W, \lambda, A}$. Due to this homothetic transformation, we can recover the volumes of $\widetilde{\mathcal{D}}_{C W, A}$ and $\widetilde{\mathcal{D}}_{C W, \lambda, A}$ from the volumes of $\mathcal{D}_{C W, A}$ and $\mathcal{D}_{C W, \lambda, A}$ by noting that $t$ varies from 0 to 1 . That is

$$
\operatorname{vol}\left(\widetilde{\mathcal{D}}_{C W, A}\right)=\int_{0}^{1} t^{11} \operatorname{vol}\left(\mathcal{D}_{C W, A}\right) d t=\frac{\operatorname{vol}\left(\mathcal{D}_{C W, A}\right)}{12}
$$

and

$$
\operatorname{vol}\left(\widetilde{\mathcal{D}}_{C W, \lambda, A}\right)=\int_{0}^{1} t^{11} \operatorname{vol}\left(\mathcal{D}_{C W, \lambda, A}\right) d t=\frac{\operatorname{vol}\left(\mathcal{D}_{C W, \lambda, A}\right)}{12} .
$$

Since the Condorcet efficiency of the weighted scoring rule $F_{\lambda}$ over $\widetilde{\mathcal{D}}$ is the ratio $\frac{\operatorname{vol}\left(\widetilde{\mathcal{D}}_{C W, \lambda, A}\right)}{\operatorname{vol}\left(\widetilde{\mathcal{D}}_{C W, A}\right)}$, the equations (9) and (10) imply the following result. 
Proposition 1 For all $\lambda \in[0,1]$, the IAC-based Condorcet efficiencies of the weighted scoring rule associated with $\lambda$ with or without unconcerned voters coincide.

In other words, Proposition 1 shows that the presence of unconcerned voters does not affect the Condorcet efficiency of weighted scoring rules under the IAC assumption as the total number of voters tends to infinity. It is clear that this is also the case for all other similar voting events that can be described by linear constraints with null constant terms. However, this is not necessarily the case with other probability distributions, such as the Impartial Culture assumption; see Gehrlein and Valognes (2001) where the authors include the possibility of having unconcerned voters.

\section{Condorcet efficiency with abstention allowed}

In this section, we assume that all voters are concerned voters, individual preferences are linear orders and some voters may abstain. Out of the initial proportion $x_{j}$ of voters of type $j$, we are now expecting that $y_{j}$ voters will effectively take part in the election. Then, the participation rate can be calculated as the number of voters who will effectively take part in the election divided by the total number of voters. A voting scenario is a twofold vector $(x, y)$ where $x=\left(x_{1}, x_{2}, \ldots, x_{6}\right)$ is a voting situation on linear orders and $y=\left(y_{1}, y_{2}, \ldots, y_{6}\right)$ satisfies

$$
0 \leq y_{j} \leq x_{j} \text { for } j=1,2, \ldots, 6 .
$$

The question is, assuming that a candidate $X$ is a Condorcet winner at $x$, some voters abstain and $y$ describes the proportion of voters from each type who finally participate in the election, what is the probability that $X$ will be selected by a given weighted scoring rule? We evaluate this conditional probability over three distinct domains.

Without loss of generality, we assume that $A$ is the Condorcet winner (or the popular candidate). We refer to the first domain as the global abstention: voters from any type may abstain. The second domain is called self-confident abstention: only voters who top ranked the popular candidate may abstain. The third domain is called pessimistic abstention: only voters who prefer all other candidates to the popular candidate may abstain.

\subsection{Global abstention}

In this setting, the set of voting scenarios is the set denoted by $\mathcal{S}$ that consists of all couples $(x, y)$ such that 


$$
(\mathcal{S}):\left\{\begin{array}{r}
x_{1} \geq 0, x_{2} \geq 0, x_{3} \geq 0, x_{4} \geq 0, x_{5} \geq 0, x_{6} \geq 0 \\
x_{1}+x_{2}+x_{3}+x_{4}+x_{5}+x_{6}=1 \\
y_{1} \geq 0, y_{2} \geq 0, y_{3} \geq 0, y_{4} \geq 0, y_{5} \geq 0, y_{6} \geq 0 \\
x_{1} \geq y_{1}, x_{2} \geq y_{2}, x_{3} \geq y_{3}, x_{4} \geq y_{4}, x_{5} \geq y_{5}, x_{6} \geq y_{6}
\end{array}\right.
$$

This domain is an 11-dimensional polytope. We assume that all voting scenarios in $\mathcal{S}$ are equally probable; and we refer to this probability distribution as $I A C_{\mathcal{S}}$. Now the subset $\mathcal{S}_{C W, A}$ of $\mathcal{S}$, that consists of all voting scenarios $(x, y)$ in which $A$ is the Condorcet winner at $x$, is the polytope characterized by the constraints at (12) and the following

$$
\left\{\begin{array}{l}
x_{3}+x_{4}+x_{6}-x_{1}-x_{2}-x_{5}<0 \\
x_{5}+x_{6}+x_{4}-x_{1}-x_{2}-x_{3}<0
\end{array}\right.
$$

Its volume is $\operatorname{vol}\left(\mathcal{S}_{C W, A}\right)=\frac{79}{10218700800}$. Since the volume of $\mathcal{S}$ is $\frac{1}{11 !}$, it follows that the probability that a Condorcet winner exists in $\mathcal{S}$ under the $I A C_{\mathcal{S}}$ assumption is $\frac{\operatorname{vol}\left(\mathcal{S}_{C W, A}\right)}{\operatorname{vol}(\mathcal{S})}=0.30859$, which gives the proportion of voting situations $(x, y)$ having candidate $A$ as a Condorcet winner when the number of voters tends to infinity. Notice that using the symmetry of IAC-like assumptions with regards to candidates means that $0.30859 \times 3=0.92578$ is the proportion of voting situations $(x, y)$ having a Condorcet winner when the number of voters tends to infinity. Finally, $A$ is selected by the weighted scoring rule associated with $\lambda$ at $y$ if and only if

$$
\left\{\begin{array}{c}
(\lambda-1) y_{1}-y_{2}+(1-\lambda) y_{3}-\lambda y_{5}+y_{4}+\lambda y_{6}<0 \\
-y_{1}+(\lambda-1) y_{2}-\lambda y_{3}+\lambda y_{4}+(1-\lambda) y_{5}+y_{6}<0
\end{array}\right.
$$

The subset $\mathcal{S}_{C W, A, \lambda}$ of $\mathcal{S}$ that consists of all voting scenarios $(x, y)$ in which $A$ is the Condorcet winner at $x$ and is selected in $y$ is the polytope described by the constraints at (12), (13) and (14). Its volume is computed in order to derive the Condorcet efficiency $C E\left(\lambda, I A C_{\mathcal{S}}, \infty\right)=\frac{\operatorname{vol}\left(\mathcal{S}_{C W, A, \lambda}\right)}{\operatorname{vol}\left(\mathcal{S}_{C W, A}\right)}$ when the total number of voters tends to infinity. The results of our calculations are given as follows:

For $0 \leq \lambda \leq \frac{1}{2}, C E\left(\lambda, I A C_{\mathcal{S}}, \infty\right)=$

$$
\frac{\left(\begin{array}{l}
218700 \lambda^{21}-1174320 \lambda^{20}-4535142 \lambda^{19}+18714908 \lambda^{18}+151671536 \lambda^{17} \\
-508196052 \lambda^{16}-1757330525 \lambda^{15}+9181808848 \lambda^{14}-2416926066 \lambda^{13} \\
-55062774610 \lambda^{12}+116439091808 \lambda^{11}-3497495094 \lambda^{10}-342404967208 \lambda^{9} \\
+608347988900 \lambda^{8}-430343075808 \lambda^{7}-70310632700 \lambda^{6}+424083710296 \lambda^{5} \\
-414925509984 \lambda^{4}+222950616032 \lambda^{3}-72115069504 \lambda^{2}+13241739264 \lambda \\
-1067873280
\end{array}\right.}{204768(1-\lambda)^{3}(3 \lambda-2)^{2}(\lambda-2)^{4}(-4+5 \lambda)^{2}\left(\lambda^{2}+2 \lambda-2\right)^{3}(1+\lambda)}
$$


For $\frac{1}{2} \leq \lambda \leq 1, C E\left(\lambda, I A C_{\mathcal{S}}, \infty\right)=$

$\frac{\left(\begin{array}{l}72900 \lambda^{24}-38460 \lambda^{23}+12014686 \lambda^{22}-284507414 \lambda^{21}+2137697548 \lambda^{20} \\ -7182774684 \lambda^{19}+9390687357 \lambda^{18}+4929800229 \lambda^{17}-18153355218 \lambda^{16} \\ -26293402260 \lambda^{15}+109425128388 \lambda^{14}-115472223994 \lambda^{13}+32683701680 \lambda^{12} \\ +26299843928 \lambda^{11}-23324433021 \lambda^{10}+2405339031 \lambda^{9}+6496354764 \lambda^{8} \\ -5222096538 \lambda^{7}+2209396698 \lambda^{6}-610438788 \lambda^{5}+115276342 \lambda^{4} \\ -14795282 \lambda^{3}+1234756 \lambda^{2}-60264 \lambda+1296\end{array}\right)}{204768 \lambda^{5}(5 \lambda-1)^{2}(2-\lambda)^{3}(1+\lambda)^{4}\left(-4 \lambda+1+\lambda^{2}\right)^{3}(-1+3 \lambda)}$

Numerical results of $C E\left(\lambda, I A C_{\mathcal{S}}, \infty\right)$ are reported in Table 2 and sketched in Figure 2.

Table 2 Condorcet efficiency of weighted scoring rules with distinct abstention scenarios

\begin{tabular}{c|ccc}
\hline$\lambda$ & $\begin{array}{c}\text { Self-confident } \\
\text { abstention }\end{array}$ & $\begin{array}{c}\text { Global } \\
\text { abstention }\end{array}$ & $\begin{array}{c}\text { Pessimistic } \\
\text { abstention }\end{array}$ \\
\hline 0 & 0.4979 & 0.6366 & 0.9722 \\
0.1 & 0.5001 & 0.6427 & 0.9801 \\
0.2 & 0.5018 & 0.6481 & 0.9865 \\
0.3 & 0.5028 & 0.6522 & 0.9905 \\
0.4 & 0.5029 & 0.6541 & 0.9906 \\
0.5 & 0.5013 & 0.6521 & 0.9841 \\
0.6 & 0.4970 & 0.6435 & 0.9662 \\
0.7 & 0.4888 & 0.6268 & 0.9304 \\
0.8 & 0.4765 & 0.6023 & 0.8744 \\
0.9 & 0.4612 & 0.5718 & 0.8021 \\
1 & 0.4443 & 0.5384 & 0.7209
\end{tabular}

\subsection{Self-confident abstention}

Assume now that individual preferences are linear orders and that only voters of type $A B C$ or $A C B$ may abstain: due to some signals such as polls surveys, some of these voters may be (erroneously or not) thinking that their favorite candidate $A$ is sufficiently popular and does not especially need their votes to defeat $B$ and $C$. With a similar notation as above, the corresponding set of voting scenarios $(x, y)$ is denoted by $\mathcal{S}^{*}$ and is now 7-dimensional since we should have $y_{j}=0$ for $j=3,4,5,6$. In the same way, the set $\mathcal{S}_{C W, A}^{*}$ and $\mathcal{S}_{C W, A, \lambda}^{*}$ are simply obtained, respectively, from $\mathcal{S}_{C W, A}$ and $\mathcal{S}_{C W, A, \lambda}$ by setting $y_{j}=0$ for $j=3,4,5,6$. Finally $C E\left(\lambda, I A C_{\mathcal{S}^{*}}, \infty\right)=\frac{\operatorname{vol}\left(\mathcal{S}_{C W, A, \lambda}^{*}\right)}{\operatorname{vol}\left(\mathcal{S}_{C W, A}^{*}\right)}$ is obtained by performing a volume computation as before. Our results are described as follows: 
Fig. 2 Condorcet efficiency of weighted scoring rules with distinct abstention scenarios

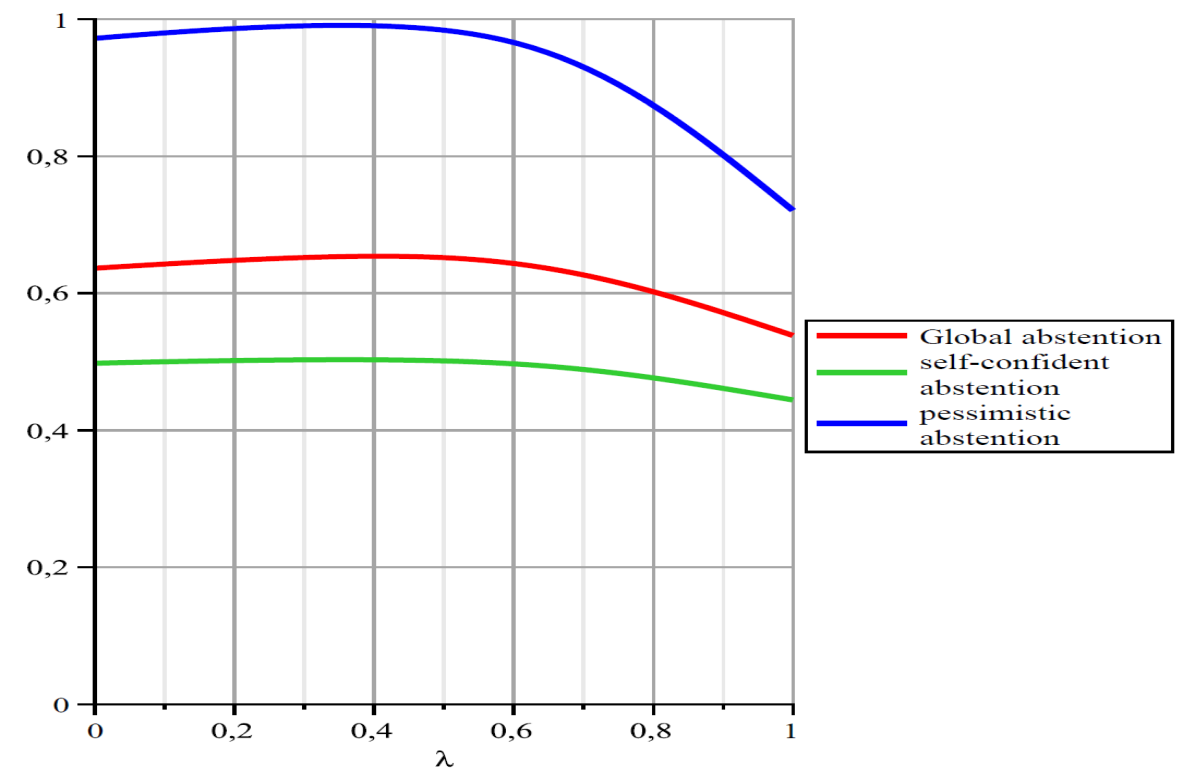

For $0 \leq \lambda \leq \frac{1}{2}, C E\left(\lambda, I A C_{\mathcal{S}^{*}}, \infty\right)=$

$\frac{\left(\begin{array}{c}34 \lambda^{13}-913 \lambda^{12}-3554 \lambda^{11}+36150 \lambda^{10}+15318 \lambda^{9} \\ -384783 \lambda^{8}+458022 \lambda^{7}+1030506 \lambda^{6}-2830398 \lambda^{5} \\ +1799251 \lambda^{4}+1181642 \lambda^{3}-2266676 \lambda^{2}+1182216 \lambda-216816\end{array}\right)}{13608(-1+\lambda)^{3}\left(\lambda^{2}+2 \lambda-2\right)^{2}(2-\lambda)^{3}(1+\lambda)}$

For $\frac{1}{2} \leq \lambda \leq 1, C E\left(\lambda, I A C_{\mathcal{S}^{*}}, \infty\right)=$

$\frac{\left(\begin{array}{c}31350 \lambda^{16}+424085 \lambda^{15}-5998520 \lambda^{14}+19077007 \lambda^{13} \\ -5835650 \lambda^{12}-52134452 \lambda^{11}+46525702 \lambda^{10}+55349687 \lambda^{9} \\ -78736170 \lambda^{8}+26324586 \lambda^{7}+8272256 \lambda^{6}-10772558 \lambda^{5} \\ +4654654 \lambda^{4}-1146839 \lambda^{3}+169090 \lambda^{2}-13868 \lambda+488\end{array}\right)}{13608(-1+3 \lambda)\left(-4 \lambda+1+\lambda^{2}\right)^{2}(1+\lambda)^{3}(5 \lambda-1)^{2} \lambda^{3}(2-\lambda)}$

Numerical results of $C E\left(\lambda, I A C_{\mathcal{S}^{*}}, \infty\right)$ are also reported in Table 2 and sketched in Figure 2. 


\subsection{Pessimistic abstention}

Finally, assume that individual preferences are linear orders and that only voters of type $B C A$ or $C B A$ may abstain: they may be (erroneously or not) feeling that $B$ and $C$ are lagging behind $A$, and that their votes for both $B$ and $C$ would be of no use. The corresponding set of voting scenarios $(x, y)$ is denoted by $\mathcal{S}^{\prime}$ and is now 7-dimensional since we should have $y_{j}=0$ for $j=1,2,3,5$. The sets $\mathcal{S}_{C W, A}^{\prime}$ and $\mathcal{S}_{C W, A, \lambda}^{\prime}$ are simply obtained, respectively, from $\mathcal{S}_{C W, A}$ and $\mathcal{S}_{C W, A, \lambda}$ by setting $y_{j}=0$ for $j=1,2,3,5$. We then get $C E\left(\lambda, I A C_{\mathcal{S}^{\prime}}, \infty\right)=\frac{\operatorname{vol}\left(\mathcal{S}_{C W, A, \lambda}^{\prime}\right)}{\operatorname{vol}\left(\mathcal{S}_{C W, A}^{\prime}\right)}$ which is obtained by performing the same volume computations as before. Our results are given as follows:

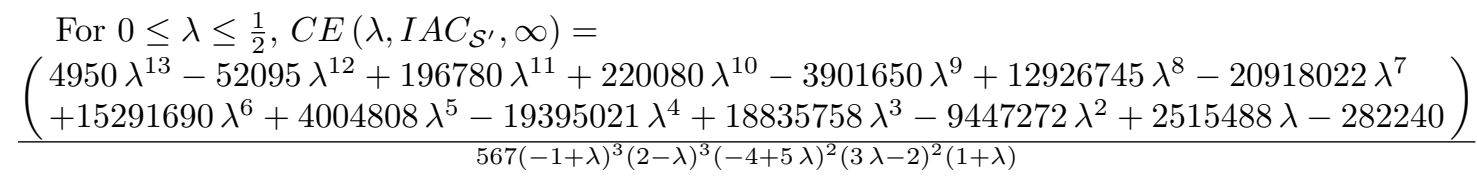

For $\frac{1}{2} \leq \lambda \leq 1, C E\left(\lambda, I A C_{\mathcal{S}^{\prime}}, \infty\right)=$ $\frac{\left(\begin{array}{l}1416 \lambda^{11}+27740 \lambda^{10}-30728 \lambda^{9}-73976 \lambda^{8}+29112 \lambda^{7} \\ +162704 \lambda^{6}-113362 \lambda^{5}+12585 \lambda^{4}+19471 \lambda^{3}-10835 \lambda^{2}+2195 \lambda-162\end{array}\right)}{2268 \lambda^{4}(1+\lambda)^{3}(2-\lambda)(-1+3 \lambda)}$

Numerical results of $C E\left(\lambda, I A C_{\mathcal{S}^{\prime}}, \infty\right)$ are also displayed in Table 2 and Figure 2.

Several lessons may be drawn from the probabilities corresponding to the three scenarios taken into account. First, it can be seen clearly that the Condorcet efficiency of the three considered scenarios exhibits the same behavior since the three curves first increase and then decrease. Every Condorcet efficiency stops rising and starts falling for a unique value $\lambda^{*}$ of $\lambda$ that maximizes the associated probability. An approximation up to four decimal places of the value of $\lambda^{*}$ maximizing the Condorcet efficiency gives $\lambda^{*} \approx 0.4074$ with $C E\left(\lambda^{*}, I A C_{\mathcal{S}}, \infty\right) \approx 0.6542$ for the global abstention domain, $\lambda^{*} \approx 0.3567$ with $C E\left(\lambda^{*}, I A C_{\mathcal{S}^{\prime}}, \infty\right) \approx 0.5030$ for the self-confident abstention scenario, and $\lambda^{*} \approx 0.3541$ with $C E\left(\lambda^{*}, I A C_{\mathcal{S}^{*}}, \infty\right) \approx 0.9912$ for the pessimistic abstention case. Third, it can be noted that on the one hand, the Condorcet efficiency remains approximately stable with regards to the value of $\lambda$ when the setting of self-confident abstention is assumed. On the other hand, the change in the Condorcet efficiency is more pronounced when the pessimistic abstention domain is considered; its value steady declines, particularly when the value of $\lambda$ exceeds 0.6 . Finally, it is worth noting that all weighted scoring rules in three-candidate elections have highest performance with respect to 
the Condorcet criterion when the pessimistic abstention domain is assumed, the self-confident abstention domain being the worst scenario.

\section{Conclusion}

Given an arbitrary weighted scoring rule for three-candidate elections, the aim of this paper has been to provide the exact limit of its Condorcet efficiency as the total number of voters tends to infinity under some IAC-like assumptions over some domains of voting situations. More exactly, we have explored the impact of observing ties and abstention on the Condorcet efficiency of the whole class of weighted scoring rules in three-candidate elections under IAC-like assumptions. Some instructive observations have emerged. First, it appears that the weighted scoring rule that maximizes the Condorcet efficiency under IAC-type assumptions depends not only on the set of observable individual preferences, but also on the behavior of voters in the election such as abstention. Second, and more importantly, the scoring rule which tends to maximize the probability of selecting the Condorcet winner, when there is one, is not the well-known Borda rule. This result has also been shown in previous studies that have been conducted in other frameworks (see for instance, Cervone et al., 2005; Lepelley et al., 2000, among others).

Many questions still remain unanswered. First, since ties and abstention have been treated separately in our framework, we believe that studying the weighted scoring rules that maximize the Condorcet efficiency, when both ties and abstention can be expressed at the same time by voters, remains a fruitful open line of research. Second, the extension of our results to multistage elimination scoring rules is also an important research direction. Under those voting rules, candidates are assigned scores according to their rank in the preferences of voters and then the candidate(s) with the lowest number of points are eliminated in each round. In this connection, other well-known voting rules widely studied in the literature can also be considered. Third, it is important to stress that the assumptions of IC and IAC have some subtle differences. For instance, results under many frameworks in the literature suggest that the Borda rule will maximize the limiting Condorcet efficiency with IC, but we have seen that it did not under IAC. As a consequence, it seems that the ways under which the voters' preferences are generated and their impact on the Condorcet efficiency of weighted scoring rules in all the scenarios considered in our paper is an important research direction to follow. Notice finally that analogous calculations would need to be done with more than three candidates, and it seems that certain new research techniques will

make this possible. Results for more than three candidates will allow us to draw more accurate conclusions.

Throughout our analysis, we have assumed that voters abstain because they think they have no chance of changing the outcome. One might as well 
consider the case of a strategic behavior where they would try to change the result in their favor: such a work would join the recent analysis of Felsenthal and Nurmi (2019) and Kamwa et al. (2018) among others, which deal with the No-show paradox under various restrictions of domains like that of the existence of the Condorcet winner.

\section{Acknowledgments}

The authors thank two anonymous reviewers for their valuable comments and suggestions. Mostapha Diss gratefully acknowledges the financial support of IDEXLYON from Université de Lyon (project INDEPTH) within the Programme Investissements d'Avenir (ANR-16-IDEX-0005).

\section{Appendix: Condorcet efficiency of standard weighted scoring rules for the case of indifference}

For $0 \leq \lambda \leq 2-\sqrt{3}, C E\left(\lambda, I A C_{\mathcal{D}}, \infty\right)=$

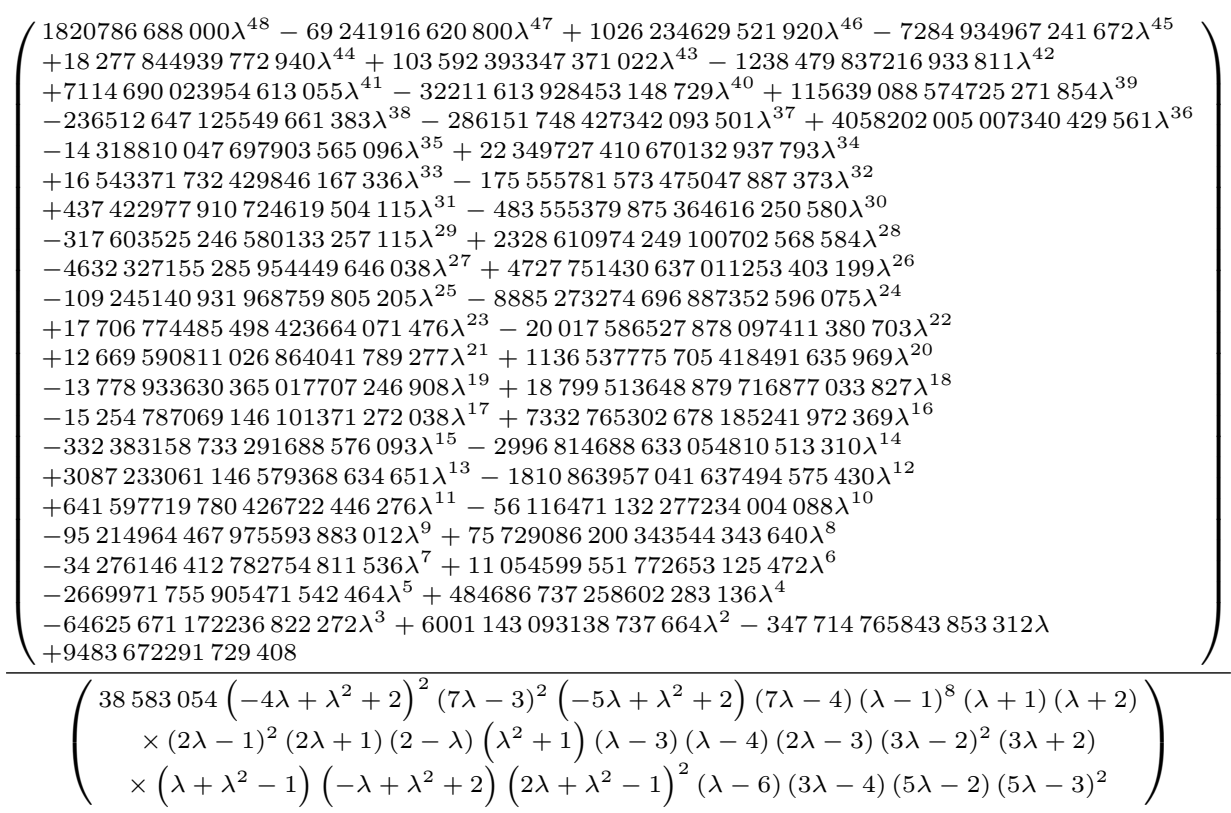

For $2-\sqrt{3} \leq \lambda \leq \frac{1}{3}, C E\left(\lambda, I A C_{\mathcal{D}}, \infty\right)=$ 


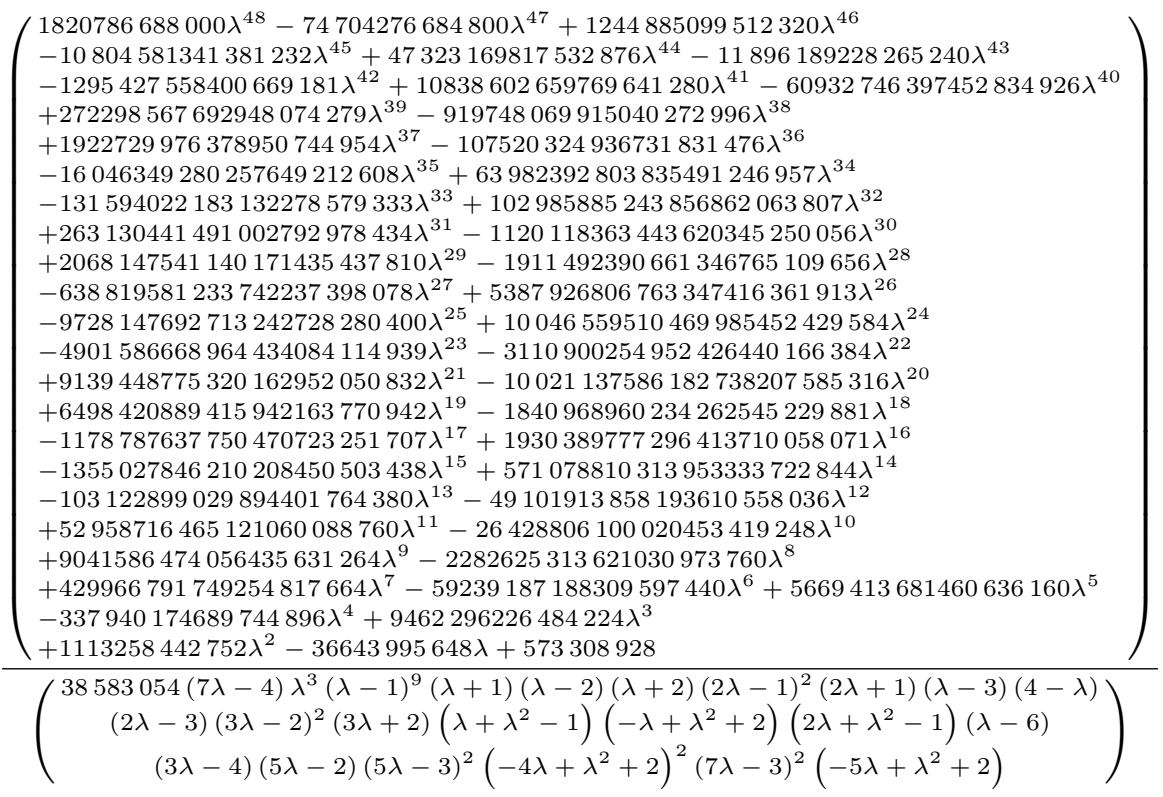

For $\frac{1}{3} \leq \lambda \leq \frac{1}{2}, C E\left(\lambda, I A C_{\mathcal{D}}, \infty\right)=$

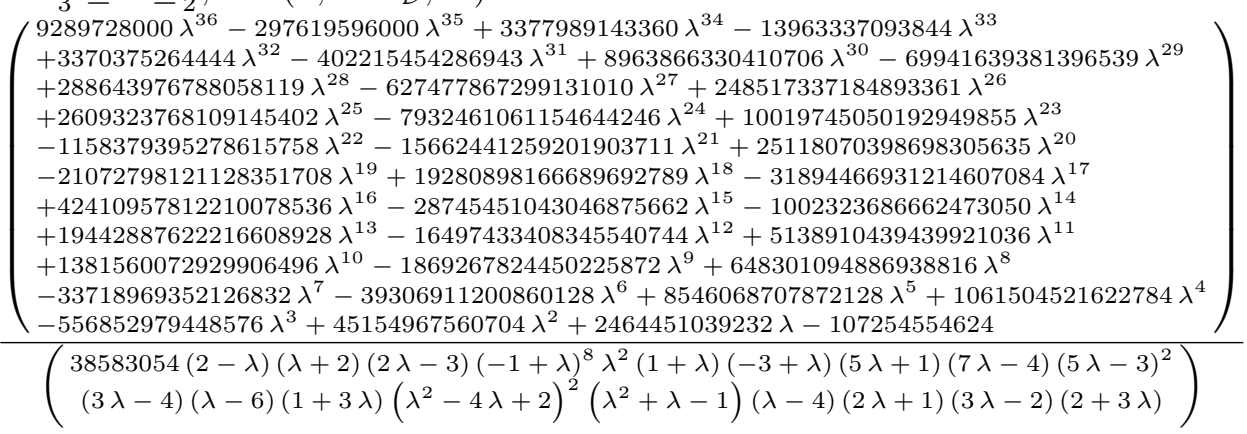

For $\frac{1}{2} \leq \lambda \leq \frac{2}{3}, C E\left(\lambda, I A C_{\mathcal{D}}, \infty\right)=$ 


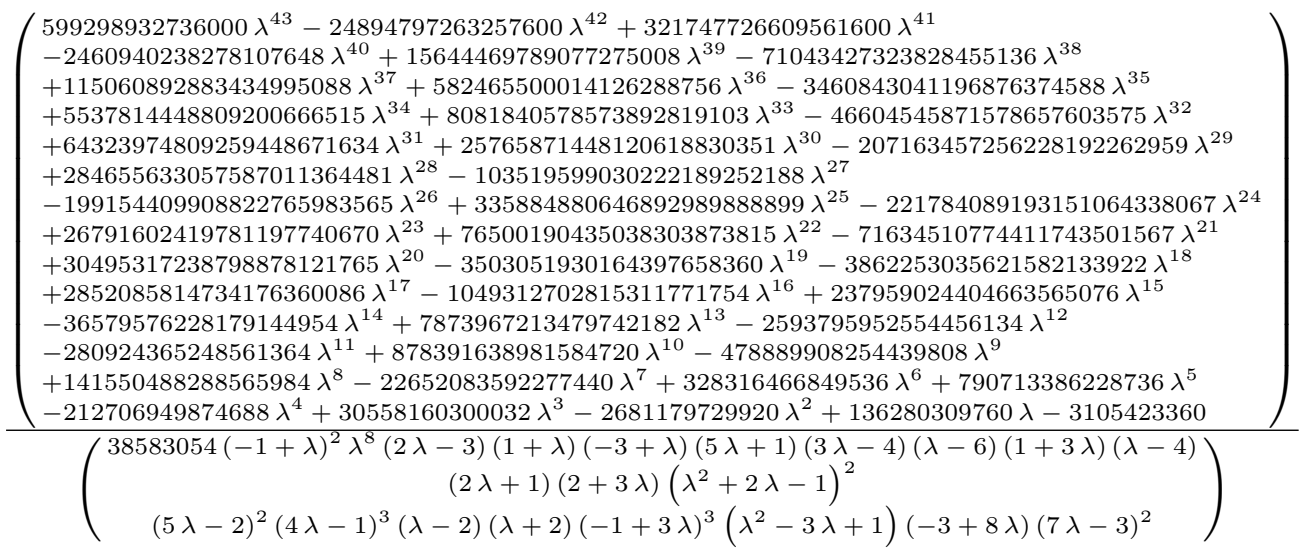

For $\frac{2}{3} \leq \lambda \leq 1, C E\left(\lambda, I A C_{\mathcal{D}}, \infty\right)=$

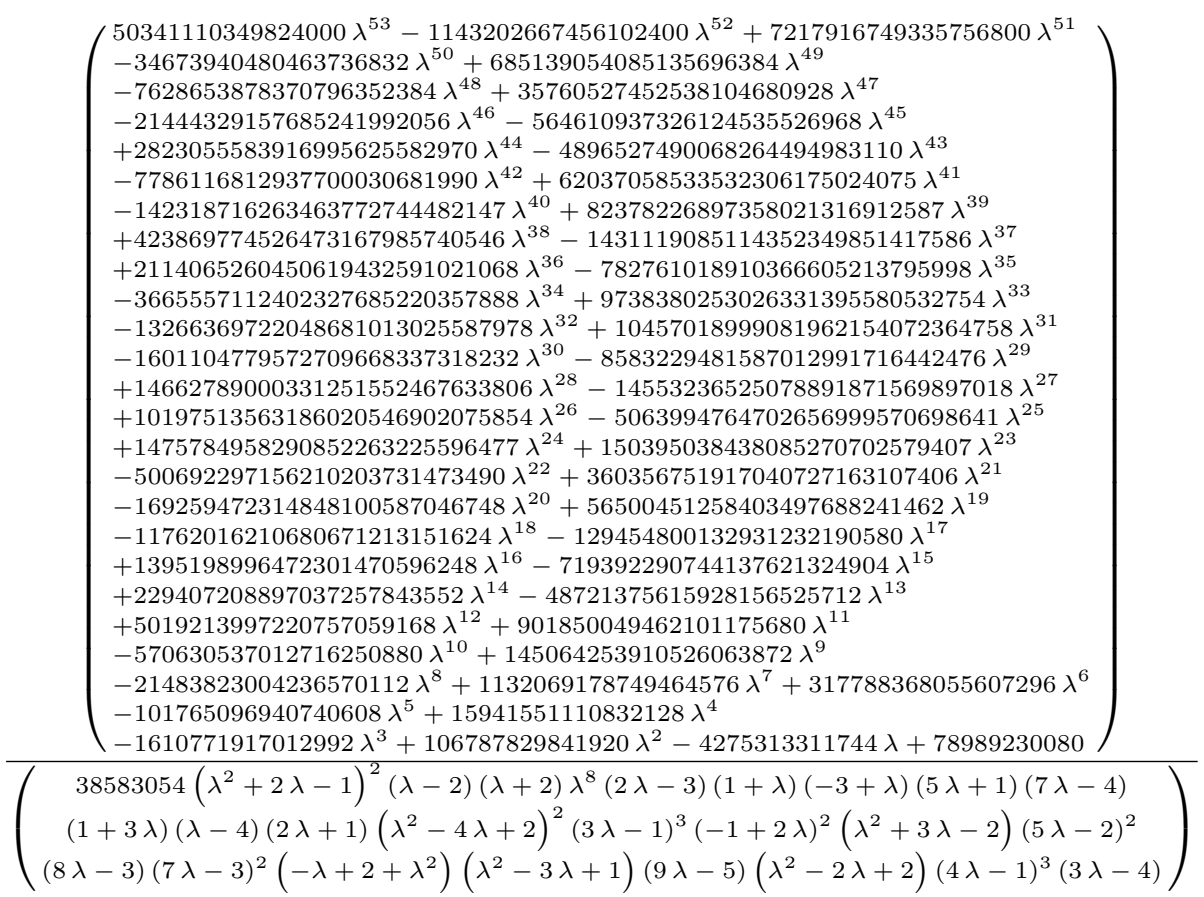

\section{References}

de Borda, J.C (1781) Mémoire sur les élections au scrutin. Histoire de

l'académie royale des sciences, pp 657-665, Paris. 
Bruns, W., \& Ichim, B. (2010) Normaliz, algorithms for affine monoids and rational cones. Journal of Algebra 324(5), 1098-1113

Bruns, W., Ichim, B., Romer, T., Sieg, R., \& Soger, C. (2017a) Normaliz: Algorithms for rational cones and affine monoids. Available at http://normaliz.uos.de.

Bruns, W., Ichim, B., \& Soger, C. (2019) Computations of volumes and Ehrhart series in four candidates elections. Forthcoming in Annals of $\mathrm{Op}$ erations Research, https://doi.org/10.1007/s1047

Bubboloni, D., Diss, M., \& Gori, M. (2019) Extensions of the Simpson voting rule to the committee selection setting. Forthcoming in Public Choice.

Cervone, D.P., Gehrlein, W.V., \& Zwicker, W.S. (2005) Which scoring rule maximizes Condorcet efficiency under IAC? Theory and Decision 2(58), $145-185$.

Condorcet, Marquis de (1785) Essai sur l'application de l'analyse à la probabilité des décisions rendues à la pluralité des voix. Paris.

Courtin, S., Martin, M., \& Moyouwou, I. (2015) The q-majority efficiency of positional rules. Theory and Decision 1(79), 31-49.

Courtin, S., Martin, M., \& Tchantcho, B. (2015) Positional rules and $q$ Condorcet consistency. Review of Economic Design 3(19), 229-245.

Diss, M., \& Doghmi, A. (2016) Multi-winner scoring election methods: Condorcet consistency and paradoxes. Public Choice 169, 97-116.

Diss, M., Kamwa, E., \& Tlidi, A. (2018) A note on the likelihood of the absolute majority paradoxes. Economics Bulletin 38(4), 1727-1734.

Diss, M., \& Gehrlein, W.V. (2015) The true impact of voting rule selection on Condorcet efficiency. Economics Bulletin 35(4), 2418-2426.

Diss, M., \& Gehrlein, W.V. (2012) Borda's paradox with weighted scoring rules. Social Choice and Welfare 38, 121-136.

Diss, M., Merlin, V., \& Valognes, F. (2010) On the Condorcet efficiency of approval voting and extended scoring rules for three alternatives. In Handbook on approval voting, Laslier, J.-F. and Sanver, R. editors, pp 255283. Springer, Berlin.

El Ouafdi, A., Lepelley, D., \& Smaoui, H. (2019) Probabilities of electoral outcomes: from three-candidate to four-candidate elections. Theory and Decision, forthcoming. DOI: https://doi.org/10.1007/s11238-019-09724-5.

Felsenthal, D.S. \& Nurmi, H. (2019) The no-show paradox under a restricted domain. Homo Oeconomicus 35(4), 277-293.

Franz, M. (2017) Convex - a maple package for convex geometry. Version 1.2 available at http://wwwhome.math.uwo.ca/mfranz/convex/.

Gehrlein, W.V. (1983) Condorcet's paradox. Theory and Decision 2(15), 161197.

Gehrlein, W.V. (2006) Condorcet's paradox. Springer Berlin Heidelberg.

Gehrlein, W.V., \& Fishburn, P.C. (1976) Condorcet's paradox and anonymous preference profiles. Public Choice 1(26), 1-18.

Gehrlein, W.V., \& Fishburn, P.C. (1978) The effects of abstentions on election outcomes. Public Choice 2(33), 69-82. 
Gehrlein, W.V., \& Fishburn, P.C. (1979) Effects of abstentions on voting procedures in three-candidate elections. Behavioral Science 5(24), 346-354.

Gehrlein, W.V., \& Lepelley, D. (2011) Voting paradoxes and group coherence: the Condorcet efficiency of voting rules. Springer Science, Business Media.

Gehrlein, W.V., \& Lepelley, D. (2015) The Condorcet efficiency advantage that voter indifference gives to approval voting over some other voting rules. Group Decision and Negotiation 2(24), 243-269.

Gehrlein, W.V., \& Lepelley, D. (2017) Elections, voting rules and paradoxical outcomes. Berlin: Springer.

Gehrlein, W.V., \& Lepelley, D. (2020) Analyzing the probability of election outcomes with abstentions. In Evaluating Voting Systems with Probability Models, Essays by and in honor of William Gehrlein and Dominique Lepelley. Diss, M. and Merlin, V. editors, pp ??-??. Springer, Berlin.

Gehrlein, W.V., \& Valognes, F. (2001) Condorcet efficiency: A preference for indifference. Social Choice and Welfare 1(18), 193-205.

Kamwa, E. (2019a) On the likelihood of the Borda effect: the overall probabilities for general weighted scoring rules and scoring runoff rules. Group Decision and Negotiation 28(3), 519-541.

Kamwa, E. (2019b) Condorcet efficiency of the preference approval voting and the probability of selecting the Condorcet loser. Forthcoming in Theory and Decision, 87(3), 299-320.

Kamwa, E., \& Moyouwou, I. (2020) Susceptibility to manipulation by sincere truncation: the case of scoring rules and scoring runoff systems. In Evaluating Voting Systems with Probability Models, Essays by and in honor of William Gehrlein and Dominique Lepelley. Diss, M. and Merlin, V. editors, pp ??-??. Springer, Berlin.

Kamwa, E., Merlin, V., \& Top, F. (2018) Scoring Runoff Rules, Single-peaked Preferences and Paradoxes of Variable Electorate. Working paper, CREM University of Caen.

Lepelley, D., Moyouwou, I., \& Smaoui, H. (2018) Monotonicity paradoxes in three-candidate elections using scoring elimination rules. Social Choice and Welfare 50(1), 1-33.

Lepelley, D., Louichi, A., \& Smaoui, H. (2008) On Ehrhart polynomials and probability calculations in voting theory. Social Choice and Welfare 30(3), 363-383.

Lepelley, D., Pierron, P., \& Valognes, F. (2000) Scoring rules, Condorcet efficiency, and social homogeneity. Theory and Decision 49, 175-196.

Lepelley, D., \& Smaoui, H. (2019) Comparing two ways for eliminating candidates in three-alternative elections using sequential scoring rules. Group Decision and Negotiation 28(4), 787-804.

Merlin, V., \& Valognes, F. (2004) The impact of indifferent voters on the likelihood of some voting paradoxes. Mathematical Social Sciences 3(48), 343-361. 
Moyouwou, I., \& Tchantcho, H. (2017) Asymptotic vulnerability of positional voting rules to coalitional manipulation. Mathematical Social Sciences 89, $70-82$. 